\title{
Screening of the NIH Clinical Collection for inhibitors of HIV-1 integrase activity
}

\section{Shaakira Abrahams $s^{1,2}$ Salerwe Mosebi ${ }^{1}$ iD Muhammed Q. Fish',2 Maria A. Papathanasopoulos ${ }^{2}$ Raymond Hewer ${ }^{3}$ iD}

\section{AFFILIATIONS:}

${ }^{1}$ Centre for Metal-Based Drug Discovery, Advanced Materials Division, Johannesburg, South Africa

2Department of Molecular Medicine and Haematology, University of the Witwatersrand Medical School, Johannesburg South Africa

${ }^{3}$ Discipline of Biochemistry, University of KwaZulu-Natal, Pietermaritzburg, South Africa

\section{CORRESPONDENCE TO: Raymond Hewer}

\section{EMAIL:}

hewerr@ukzn.ac.za

\section{DATES:}

Received: 21 Sep. 2017

Accepted: 12 Nov. 2017

Published: 27 Mar. 2018

\section{KEYWORDS:}

drug repurposing; strand transfer; pan-assay interference compounds; PAINS

\section{HOW TO CITE:}

Abrahams S, Mosebi S, Fish $M Q$, Papathanasopoulos MA Hewer R. Screening of the NIH Clinical Collection for inhibitors of HIV-1 integrase activity. S Afr J Sci. 2018;114(3/4) Art. \#2017-0324, 5 pages. http://dx.doi.org/10.17159/ sajs.2018/20170324

\section{ARTICLE INCLUDES: \\ $\times$ Supplementary material \\ $\times$ Data set}

\section{FUNDING:}

National Research Foundation (South Africa)

\section{(c) 2018. The Author(s).} Published under a Creative Commons Attribution Licence.
Drug repurposing offers a validated approach to reduce drug attrition within the drug discovery and development pipeline through the application of known drugs and drug candidates to treat new indications. Full exploitation of this strategy necessitates the screening of a vast number of molecules against an extensive number of diseases of high burden or unmet need and the subsequent dissemination of the findings. In order to contribute to endeavours within this field, we screened the 727 compounds comprising the US National Institutes of Health (NIH) Clinical Collection through an HIV-1 (human immunodeficiency virus type 1) integrase stand transfer inhibition assay on an automated scintillation proximity assay platform. Only two compounds were identified within the initial screen, with cefixime trihydrate and epigallocatechin gallate found to reduce integrase strand transfer activity at $\mathrm{IC}_{50}$ values of $6.03 \pm 1.29 \mu \mathrm{M}$ and $9.57 \pm 1.62 \mu \mathrm{M}$, respectively. However, both cefixime trihydrate and epigallocatechin gallate retained their low micromolar inhibitory activity when tested against a raltegravir-resistant integrase double mutant $\left(\mathrm{FCIC}_{50}\right.$ values of 0.83 and 0.06 , respectively), were ineffective in an orthogonal strand transfer ELISA $(<30 \%$ inhibition at $100 \mu \mathrm{M})$ and produced negligible selectivity index values $(<1)$ in vitro. While no useful inhibitors of HIV-1 integrase strand transfer activity were found within the NIH Clinical Collection, the identification of two assay-disrupting molecules demonstrates the importance of consideration of non-specific inhibitors in drug repurposing screens.

\section{Significance:}

- This study is the first to screen the US NIH Clinical Collection for potential HIV-1 integrase inhibitors.

- $\quad$ The pervasive nature of promiscuous inhibitors is emphasised.

\section{Introduction}

Early-stage drug discovery fulfils a critical role within the broader drug discovery process and the entire drug discovery and development pipeline. Early-stage drug discovery is typically - but not always - undertaken following target identification and validation, and involves the screening of compounds with the intent purpose of identifying compounds with promising activity (HIT compounds) that can then be developed further (into LEAD compounds) within the drug discovery phase. Early-stage drug discovery activities can range from the evaluation of a limited set of compounds, typically selected through rational drug design methodologies, to the assessment of large compound libraries through high throughput screening (HTS; defined as the screening of $>10000$ compounds per day) and even ultra-HTS ( $\mu \mathrm{HTS}$; defined as the screening of $>100000$ compounds per day) operations. Owing to the sheer number of compounds screened, early-stage drug discovery ostensibly carries the highest failure rate and, accordingly, the highest risk of all activities within the pipeline. However, the true bottleneck to success in the broader drug discovery and development process lies less with the quantity of compounds identified as HITS during screening and more with the quality of these compounds and their suitability as drug candidates. Specifically, the highest cause for compound attrition in the pipeline, by far, is attributed to non-clinical toxicity which accounts for the termination of $>40 \%$ of all compounds from the drug discovery and development pipeline. ${ }^{1}$

To mitigate the potential significant financial loss resulting from compound failures, in particular the high cost of late-stage failures, most pharmaceutical organisations adopt the 'fail early, fail cheap' paradigm. To support this approach, researchers aim to recognise ADMET-related issues through an ever-increasing number of tests undertaken at progressively earlier stages of the pipeline. Equally, findings from these tests have been retrospectively accumulated to delineate physiochemical properties (i.e. LogP, LogD, molecular weight, aromatic rings, rotatable bonds, polar surface area, etc.) that influence drug-likeness and then subsequently collated into 'rules of thumb' (such as the Lipinski rule of five, the rule of three and many other variations and extensions) and property prediction software programs. Application of these predictive models has allowed for the identification and judicious removal of non-favourable compounds either following screening or directly from the physical compound library prior to screening. While immeasurably useful, these tools have not proven infallible as evinced through a recent study of 812 failed compounds (oral development candidates from four different major pharmaceutical companies) that could draw no correlation between non-clinical toxicology failure and physiochemical properties. ${ }^{1}$ Similarly, a subset of compounds eliciting growing interest because of their subversive effects in drug discovery efforts are promiscuous inhibitors ${ }^{2,3}$ or pan-assay interference compounds (PAINS) ${ }^{4}$. These compounds yield convincing falsepositive results in biological assays and significant efforts have been undertaken to identify them and ultimately remove them from screening libraries. ${ }^{4}$ Broadly categorised and inclusive of several classes of compounds with varying mechanisms of action (i.e. aggregate-inducing compounds, redox-cyclers, covalent modifiers, metal complexes), these compounds do not readily lend themselves to predictive algorithms. Nonetheless, databases of existing PAINS highlight common structures (i.e. flavonoids, quinones, rhodamines) and some can also be searched for similarity.

Of other approaches aimed at minimising compound attrition, the concept of drug repurposing (or drug repositioning) has drawn significant interest. Herein, the underlying principle is the evaluation of clinically approved drugs or 
late-stage clinical trial failures (all off-patent or generics) as diseasemodifying agents in therapeutic areas other than the one for which they were designed or proved effective. The main appeal of this approach is the decreased risk of failure arising from safety issues while the extensive prior development allows for a quicker transitioning through the pipeline (up to $60 \%$ ) with reduced costs (up to $40 \%$ ). If successful, the drug can be granted patent protection on grounds of a new application or new formulation. The classic example of drug repositioning is Viagra ${ }^{\circledR}$ - the blockbuster erectile dysfunction drug from Pfizer which first served as an angina medication under the name Sildenafil. Numerous other examples exist, including azidothydimine (the cancer turned anti-HIV drug), ropinirole (a dual Parkinson's and restless legs syndrome treatment) and Rogaine ${ }^{\circledast}$ (a hair-loss drug repurposed from a blood pressure drug from a failed ulcer candidate), to mention but a few. The scientific merit of the concept has driven growth in its popularity as is clearly evident through interest from major pharmaceutical companies, the growth in focused start-up companies, the rise in related literature and a recently launched journal (Drug Repurposing, Rescue and Repositioning) with dedicated content.

Applying the drug repurposing approach to the field of HIV drug discovery has been previously described. While the treatment options for HIV-1 are formidable - both in the number of antiretroviral agents approved and the efficacy of combination therapy - the absence of an effective therapeutic vaccine or cure and the persistent threat of antiretroviral drug resistance has substantiated the continued exploration for novel inhibitors. In this study, we sought to identify an existing drug with activity against HIV-1 integrase (IN) - a virally encoded enzyme that catalyses the integration of viral DNA into the host chromosome. For this purpose we screened the US National Institutes of Health (NIH) Clinical Collection (NCC), which is a 727 small-molecule library of FDA-approved and late-stage candidates that has been previously explored for proteasome stimulators ${ }^{5}$ and coronavirus inhibitors ${ }^{6}$ but not, to the best of our knowledge, for HIV-1 IN inhibition. The NCC library was screened by means of an automated process through an HIV-1 IN strand-transfer (ST) inhibition scintillation proximity assay (SPA) in order to identify novel catalytic IN inhibitors.

\section{Methods}

\section{Expression and purification of recombinant HIV-1 integrase}

The reagent pINSD.His (Cat. \#2957) was obtained through the NIH AIDS Reagent Program, Division of AIDS, NIAID, NIH, from Dr Robert Craigie. ${ }^{7,8}$ Recombinant His-tagged HIV-1 IN was expressed and purified as previously described. ${ }^{9}$ Briefly, wild-type HIV-1 IN was overexpressed in E. coli BL21 (DE3) bacterial cells using the NL4-3 histidine (HIS)tagged HIV-1 IN coding sequence, pINSD, cloned into pET15B (Merck Millipore, Darmstadt, Germany). Cells were grown to logarithmic phase in Luria-Bertani medium and induced with $1 \mathrm{mM}$ isopropyl-thiogalactoside. The recombinantly expressed protein was purified through affinity chromatography using a nickel (Ni)-affinity column and buffer exchanged into storage buffer (20 mM HEPES pH 7.2, $1 \mathrm{M} \mathrm{NaCl}, 4 \mathrm{mM}$ EDTA, 2 mM dithiothreitol and 50\% glycerol) using a PD-10 Sephadex column (GE Healthcare, Buckinghamshire, UK). The expression and purification of the HIV-1 IN was confirmed through SDS-PAGE and subsequent Western blot analysis. Similarly, recombinant IN which incorporated raltegravir-resistant mutations, $\mathrm{IN}_{0148 \mathrm{H} / 140 \mathrm{~S}}$, was prepared. Briefly, the pINSD. His plasmid was used as a template for mutagenesis with the QuickChange Lightning Site Directed Mutagenesis kit (Agilent Technologies, Santa Clara, CA, USA). Raltegravir-resistant mutations were inserted into the pINSD. His backbone and included Q148H/ G140S substitutions.

\section{Radiolabelling of target DNA for scintillation proximity assays}

Annealed oligonucleotides for target DNA (tDNA), T 56-S (AAAAGGAGGAGAAGGAAAGGAGAGAGAGCGAATTAGCCCTTGGTC) and T 56-A (AAAAGGA GGAGAAGGAAAGGAGAGAGAGGACCAAGGGCTAATTCG) oligonucleotides (Inqaba Biotech, Pretoria, South Africa), were radiolabelled with ${ }^{3} \mathrm{H}-\mathrm{dCTP}$ and ${ }^{3} \mathrm{H}-\mathrm{dTTP}$ (AEC Amersham, Johannesburg, South Africa) by filling in the 5'-overhangs according to the Fermentas Klenow fragment DNA labelling kit instructions (ThermoFisher Scientific, Waltham, MA, USA). Unincorporated nucleotides were removed from the radiolabelled tDNA using the QIAquick nucleotide removal kit (Qiagen, Hilden, Germany).

\section{HIV-1 integrase strand transfer scintillation proximity assay}

The SPA was carried out as previously described ${ }^{10}$ and adapted to an automated platform on a Hamilton Starlet robotic system (Hamilton, Bonaduz, Switzerland). Briefly, a 10x reaction buffer was prepared containing $200 \mathrm{mM}$ HEPES (pH 7.5), $300 \mathrm{mM} \mathrm{NaCl}, 50 \mathrm{mM}$ dithiothreitol and $0.5 \%$ Igepal (nonidet-P40). Polyvinyltoluene streptavidin-coated scintillation beads (GE Healthcare Sciences, Marlborough, MA, USA) were reconstituted in $1 \times$ reaction buffer at a final concentration of $10 \mathrm{mg} /$ $\mathrm{mL}$. Biotinylated donor DNA (dDNA) was added at a final concentration of $500 \mathrm{nM}$ and rocked at room temperature for $1 \mathrm{~h}$. The bead suspension was washed twice with $1 \times$ reaction buffer and centrifuged at $1000 \times g$ for $5 \mathrm{~min}$. The pellet was resuspended at $2 \mathrm{mg} / \mathrm{mL}$ in $2 x$ reaction buffer to which recombinant IN (wild-type or Q148H/G140S mutant) was added at a final concentration of $1 \mu \mathrm{M}$ and rocked at room temperature for 30 min. The final SPA reactions comprised, per well: $1 \mathrm{mg} / \mathrm{mL}$ SPA beaddDNA-IN complex with 8 to 10 test compounds at $10 \mu \mathrm{M}$ each for singledose experiments or concentrations ranging from 100 to $0.78 \mu \mathrm{M}$ for dose-response experiments (substituted with DMSO buffer solution for blank control). This reaction mixture was incubated at $22^{\circ} \mathrm{C}$ for 30 min whilst shaking gently. The reactions were initiated by adding $500 \mathrm{nM}^{3} \mathrm{H}-$ tDNA to each well at a final concentration of $50 \mathrm{nM}$ and incubated at 37 ${ }^{\circ} \mathrm{C}$ shaking for 90 min before the enzymatic reaction was stopped using $62 \mathrm{mM}$ EDTA. The reaction product formation was measured using the Top Count Scintillation Counter NXT (Perkin Elmer, Waltham, MA, USA). Percentage inhibition was determined for single-dose experiments while $\mathrm{IC}_{50}$ values were determined as the compound concentration required to reduce HIV-1 recombinant IN ST activity by $50 \%$ and calculated using OriginPro 8.0 software (Origin Lab Corporation, Northampton, MA, USA). All inhibition values are the average of at least triplicate experiments.

\section{HIV-1 integrase strand transfer enzyme-linked immunosorbent} assay

The HIV-1 IN strand transfer inhibition enzyme-linked immunosorbent assay (ELISA) was adapted from previously described methods. ${ }^{9,11}$ Briefly, 0.15 $\mu \mathrm{M}$ double-stranded biotinylated dDNA (5'-biotin-ACCCTTTTAGTCAGTGT GGAAAATCTCTAGCA-3' and 5'-ACTGCTAGAGATTTTCCACACTGACTAA AAG-3') was added to the wells of streptavidin-coated 96-well microtitre plates (R\&D Systems, Minneapolis, MN, USA). Following incubation at room temperature for 60 min and a stringent wash step, $1 \mu \mathrm{M}$ purified recombinant HIV-1 subtype B IN (in the presence of $\mathrm{MgCl}_{2}$ ) was assembled onto the pre-processed dDNA through incubation for $30 \mathrm{~min}$ at $22{ }^{\circ} \mathrm{C}$. Following a wash step, the test compounds were titrated into individual wells at a final concentration of $100 \mu \mathrm{M}$ for single-dose evaluation or concentrations ranging from 100 to $0.78 \mu \mathrm{M}$ for dose-response experiments. The microtitre plates were incubated for $30 \mathrm{~min}$ at $37^{\circ} \mathrm{C}$, washed and the strand transfer reaction was initiated through the addition of $0.25 \mu \mathrm{M}$ double-stranded FITC-labelled target DNA (5'-TGACCAAGGGCTAATTCACT-FITC-3' and 5'-AGTGAATTAGCCCTTGGTCA-FITC-3') in Hepes buffer containing $\mathrm{MgCl}_{2}$ and $\mathrm{MnCl}_{2}$. After an incubation period of $60 \mathrm{~min}$ at $37^{\circ} \mathrm{C}$, the plates were washed as before and an alkaline phosphatase conjugated anti-FITC secondary antibody (Sigma-Aldrich, St Louis, MO, USA) was added. Finally, the plates were washed and substrate (BluePhos, KPL, Gaithersburg, MD, USA) was added to allow for detection at $620 \mathrm{~nm}$ using a microplate reader (xMark ${ }^{\mathrm{TM}}$, Bio-Rad, Hercules, CA, USA). Percentage inhibition was determined for single-dose experiments while $\mathrm{IC}_{50}$ values were determined as the compound concentration required to reduce HIV-1 recombinant IN ST activity by $50 \%$ and calculated using OriginPro 8.0 software (Origin Lab Corporation). All inhibition values are the average of at least triplicate experiments.

\section{Cytotoxicity assays}

The reagent MT-4 (Cat. \#120) was obtained through the NIH AIDS Reagent Program, Division of AIDS, NIAID, NIH, from Dr Douglas Richman. ${ }^{12-14}$ The cytotoxicity assay was performed as per standard methods and as described previously. ${ }^{9,15}$ Briefly, MT-4 cells were plated in 96-well microtitre plates at $3.0 \times 10^{5}$ cells $/ \mathrm{mL}$ and allowed to stabilise 
for $2 \mathrm{~h}$ at $37^{\circ} \mathrm{C}$ and $5 \% \mathrm{CO}_{2}$. Thereafter, test compounds were added to the plate through twofold serial dilution to allow for eight final compound concentrations ranging from 200 to $1.56 \mu \mathrm{M}$ in a total volume of $200 \mu \mathrm{L} /$ well. The cells and compounds were then incubated for $96 \mathrm{~h}$ at $37^{\circ} \mathrm{C}$ and $5 \% \mathrm{CO}_{2}$. To each well, $20 \mu \mathrm{L}$ CellTiter 96 Aqueous One Solution (Promega, Madison, WI, USA) was added. The plates were incubated for $4 \mathrm{~h}$ and absorbance was read at $490 \mathrm{~nm}$ on a multiplate reader (xMark, Bio-Rad). $\mathrm{CC}_{50}$ values were determined as the concentration of the test compound required to reduce the cell viability by $50 \%$ and were calculated using OriginPro 8.0 software (Origin Lab Corporation). The values obtained are averages of at least three separate experiments.

\section{Antiviral activity}

To determine antiviral activity, $50 \mu \mathrm{L} \mathrm{HIV-1}{ }_{\text {NL4-3 }}$ virus was added to $3.0 \times 10^{5} \mathrm{MT}-4$ cells $/ \mathrm{mL}$ at a multiplicity of infection of 0.1 and the mixture was spinoculated at $3000 \times \mathrm{g}$ for $90 \mathrm{~min}$. After washing off unbound virus, cells were plated in 96 -well microtitre plates at $100 \mu \mathrm{L} /$ well and allowed to stabilise for $1 \mathrm{~h}$ at $37{ }^{\circ} \mathrm{C}$ and $5 \% \mathrm{CO}_{2}$. Thereafter, test compounds were added to the plate through twofold serial dilution to allow for eight final compound concentrations ranging from 200 to $1.56 \mu \mathrm{M}$ in a total volume of $200 \mu \mathrm{L} /$ well. The cells and compounds were then incubated for $96 \mathrm{~h}$ at $37^{\circ} \mathrm{C}$ and $5 \% \mathrm{CO}_{2}$. Cell-free supernatants were collected from each well, and p24 concentration was determined using the Vironostika HIV-1/2 p24 Antigen ELISA (bioMerieux, Marcy-I 'Etoile, France) as per manufacturer's instructions. $\mathrm{EC}_{50}$ values were determined as the concentration of the test compound required to reduce p24 concentration by $50 \%$ and were calculated using OriginPro 8.0 software (Origin Lab Corporation). The values obtained are averages of at least three separate experiments. Selectivity index (SI) values were calculated as the ratio of $\mathrm{CC}_{50} / \mathrm{EC}_{50}$.

\section{Results and discussion}

\section{Inhibition of recombinant integrase strand transfer activity}

To begin the screening process, all 727 small molecules within the NCC library were pooled in an orthogonal manner into 171 pools; each pool comprised a combination of 8 or 10 different compounds with each compound present in two pools. The pools were then screened through an automated SPA, in triplicate, with each compound evaluated at a final single-dose concentration of $10 \mu \mathrm{M}$. Initially, 24 pools were found to be affected by colour-quenching which was only resolved through the identification and removal of 12 coloured compounds. On completion of the screening process, four pools were found to reduce recombinant $I \mathrm{~N}_{\text {WT }}$ ST activity by the pre-defined minimum cut-off of $\geq 50 \%$, indicating the presence of two active compounds. Through cross-referencing, the two common compounds were identified as cefixime trihydrate (CEF) and epigallocatechin gallate (EGCG). CEF, a third-generation orally administered cephalosporin is a potent, broad-spectrum bactericidal. Like other cephalosporins, CEF possesses a dihydrothiazine ring fused to a beta-lactam ring and derives its therapeutic effect through inhibition of cell-wall synthesis by disruption of the transpeptidation process. Modulation of HIV-1 replication by CEF has not been previously described in the literature; however, cephalosporin oligonucleotides and monocyclic $\beta$-lactams have been reported as HIV-1 protease inhibitors. EGCG is a polyphenolic acid ester that has been proclaimed as an effective agent for an exceedingly broad range of diseases (including HIV-1 through several distinct mechanisms of action) despite its welldocumented promiscuous nature. ${ }^{4,16} \mathrm{~A}$ search of the US NIH registry and results database of clinical studies revealed 92 clinical trials in varying stages of completion (from recruiting to completed) examining EGCG as treatment for 276 different clinical conditions. ${ }^{17}$

In our experience, the HIV-1 integrase ST SPA is a robust assay that yields a low number of HIT compounds per screen. The inclusion of detergent within the protocol design serves, perhaps inadvertently, to reduce the identification of false positive responses caused by promiscuous molecules. We therefore opted to continue investigating EGCG to verify a non-specific mechanism, and as such, both CEF and
EGCG were subjected to dose-response evaluation within the previously described SPA. Here we established $\mathrm{IC}_{50}$ values of $6.03 \pm 1.29 \mu \mathrm{M}$ and $9.57 \pm 1.62 \mu \mathrm{M}$ for CEF and EGCG, respectively (Table 1) with steep slopes observed for both compounds (Hill slope $>1$ ). In comparison, raltegravir, the first-in-class HIV-1 integrase drug marketed as Isentress ${ }^{\circledR}$, inhibited recombinant $\mathbb{I N}_{\text {WT }}$ activity by $92 \pm 5 \%$ at a singledose concentration of $10 \mu \mathrm{M}$ and produced an $\mathrm{IC}_{50}$ of $9.98 \pm 0.83 \mathrm{nM}$ (Table 1) with a Hill slope of 1 . Thereafter, the compounds were tested for inhibitory activity of the raltegravir-resistant IN $_{0148 H / G 140 S}$ double-mutant. ${ }^{18}$ Replication capacity of this double mutant was reduced to $59 \%$ of $\mathbb{I N}_{\text {WT }}$ and, as anticipated, ${ }^{19}$ raltegravir was significantly less effective against this mutant $(p<0.01)$ as reflected by a fold change in $\mathrm{IC}_{50}\left(\mathrm{FCIC}_{50}\right)$ value of 214 (Table 1). On the contrary, the two identified compounds retained their micromolar inhibitory activity against the raltegravirresistant double mutant with $\mathrm{FCIC}_{50}$ values of 0.83 and 0.06 calculated for CEF and EGCG, respectively (Table 1). As a further direct evaluation of activity, the compounds were tested for ST inhibition within an HIV-1 IN ST assay based on an ELISA platform. As a true, indisputable IN ST inhibitor, raltegravir efficiently inhibited $\mathrm{IN}_{\mathrm{WT}}$ in this orthogonal assay to the same degree as that observed within the SPA-based assay $\left(\mathrm{IC}_{50}=\right.$ $10.25 \pm 0.75 \mathrm{nM}$ ) while dose-response evaluations were not warranted for CEF and EGCG as both proved ineffective inhibitors at a high singledose concentration $(<30 \%$ inhibition at $100 \mu \mathrm{M}$; Table 1). Up to this point, the behaviour of CEF and EGCG strongly supported a nonspecific role for both molecules through a related mechanism of action that disrupted the SPA-based platform. This observation subsequently led us to the work of another group that speculated a non-specific mechanism for CEF within an SPA - in this case as an inhibitor of both $\mathrm{NADH}$ dehydrogenase (at an $\mathrm{IC}_{50} \sim 8 \mu \mathrm{M}$ ) as well as MurG (at an $\mathrm{IC}_{50}$ $\sim 16 \mu \mathrm{M})^{20}$

\section{In-vitro evaluation}

EGCG and CEF were both evaluated for toxicity within the MT-4 mammalian cell line. While EGCG yielded a $\mathrm{CC}_{50}$ of $23 \mu \mathrm{M}$, CEF was not found to be toxic within the limits of the assay $(\geq 200 \mu \mathrm{M})$. Thereafter, inhibition of HIV-1 replication by CEF and EGCG was evaluated in vitro in the MT-4 cell line following infection by HIV-1 $1_{\mathrm{NL} 4-3}$. For EGCG, an $\mathrm{EC}_{50}$ of $24 \mu \mathrm{M}$ was determined through dose-response studies (Table 1). The observed viral inhibition by EGCG closely correlated the toxicity profile of the compound in the same cell line and led to a negligible SI value ( 1; Table 1). An SI value for CEF could not be determined as no observable viral inhibition was found in the cell-based HIV-1 inhibition assay up to the maximum tested compound concentration of $200 \mu \mathrm{M}$ (Table 1). As a control, raltegravir was found to inhibit HIV-1 replication within this assay with an $\mathrm{EC}_{50}$ of $16.82 \mathrm{nM}$ with no observable toxicity up to the limit of the assay $\left(\mathrm{CC}_{50} \geq 200 \mu \mathrm{M}\right.$; SI value $\geq 11890.61$ ).

\section{Conclusion}

Drug repurposing has proven successful in the past and offers a viable strategy for the discovery and development of therapeutic agents. In an endeavour to contribute to efforts in this field, we screened the NCC library to identify new inhibitors of HIV-1 integrase strand transfer activity. While no true inhibitors of HIV-1 IN ST activity were discovered, the identification of two non-specific inhibitors through our screen demonstrated that drug repurposing is not insusceptible to the presence of assay disruptors. In particular, the identification of EGCG demonstrates the invasiveness of even the most well-documented PAINS into chemical screening libraries. Furthermore, and perhaps more interestingly, the confirmation of the clinically relevant antibiotic CEF as an SPA disruptor demonstrates necessity to interrogate the action of well-characterised molecules within specific assay platforms and also supports the mandatory use of secondary or orthogonal assays to confirm inhibition. The findings from this study suggest that both EGCG and CEF disrupt the SPA through a similar non-aggregating mechanism that will be elucidated through future studies to facilitate further screening projects based on this assay platform. 
Table 1: $\quad$ Comparison of cefixime trihydrate, epigallocatechin gallate and raltegravir as inhibitors of HIV-1 integrase strand transfer activity

\begin{tabular}{|c|c|c|c|c|c|c|c|c|}
\hline \multirow[b]{3}{*}{ Compound } & \multicolumn{5}{|c|}{ HIV-1 integrase strand-transfer inhibition } & \multicolumn{3}{|c|}{ MT- 4 cell based assays } \\
\hline & \multicolumn{3}{|c|}{ Scintillation proximity assay (SPA) } & \multicolumn{2}{|c|}{ ELISA } & & & \\
\hline & $\begin{array}{c}\text { IN }_{\mathrm{WT}} \\
\mathbf{I C}_{50} \pm \mathbf{S E}(\mu \mathrm{M})\end{array}$ & $\begin{array}{c}I_{0148 \mathrm{H} / \mathrm{G} 140 \mathrm{~S}} \\
\mathrm{IC}_{50} \pm \mathrm{SE}(\boldsymbol{\mu} \mathrm{M})\end{array}$ & $\mathrm{FCIC}_{50}$ & $\%$ Inhibition & $\mathrm{IC}_{50} \pm \mathrm{SE}(\mathrm{nM})$ & $\begin{array}{c}\mathrm{CC}_{50} \pm \mathrm{SE} \\
(\mu \mathrm{M})\end{array}$ & $\begin{array}{c}\mathrm{EC}_{50} \pm \mathrm{SE} \\
(\mu \mathrm{M})\end{array}$ & SI \\
\hline Raltegravir & $9.98 \pm 0.83\left(\times 10^{-3}\right)$ & $1.50 \pm 0.50$ & 214 & $96 \%$ (at $10 \mu \mathrm{M}$ ) & $10.25 \pm 0.75$ & $>200$ & $16.82\left(\times 10^{-3}\right)$ & $\geq 11890.61$ \\
\hline Cefixime trihydrate & $6.03 \pm 1.29$ & $5.01 \pm 1.29$ & 0.83 & $\begin{array}{c}21 \% \\
\text { (at } 100 \mu \mathrm{M} \text { ) }\end{array}$ & ND & $>200$ & $>200$ & - \\
\hline Epigallocatechin ga & $9.57 \pm 1.62$ & $0.62 \pm 1.50$ & 0.06 & $\begin{array}{c}29 \% \\
\text { (at } 100 \mu \mathrm{M} \text { ) }\end{array}$ & ND & $23 \pm 1$ & $24 \pm 3$ & $\sim 1$ \\
\hline
\end{tabular}

IN wild-type HIV-1 integrase

$I C_{50}, 50 \%$ inhibitory concentration; calculated as the concentration of compound required to reduce the HIV-1 integrase strand transfer activity by $50 \%$

$S E$, Standard error; standard error of the mean for at least three separate experiments

$\mathrm{FCIC}_{50}$, fold-change in IC ${ }_{50}$; calculated as the ratio of $I \mathrm{~N}_{0148 \mathrm{H} / \mathrm{G140S}} / \mathrm{IN}_{\mathrm{WT}}$

$\mathrm{CC}_{50}, 50 \%$ cytotoxic concentration; calculated as the concentration of compound required to reduce cell viability by $50 \%$

$E C_{50}, 50 \%$ effective concentration; defined as the concentration of compound required to reduce HIV-1 replication by $50 \%$

$\mathrm{SI}$, selectivity index; calculated as the ratio of $\mathrm{CC}_{50} / E C_{50}$

ND, not done

\section{Acknowledgements}

We thank the National Research Foundation of South Africa, Mintek, the University of the Witwatersrand and the University of KwaZulu-Natal for funding and permission to publish these findings.

\section{Authors' contributions}

R.H. was responsible for the conceptualisation, methodology, data analysis, validation, critically reviewing the writing, writing revisions, student supervision, project leadership, project management and funding acquisition. S.M. was responsible for the methodology, data analysis, validation, data curation, writing revisions, student supervision and project leadership. S.A. was responsible for the methodology, data collection, data analysis, sample analysis, validation, data curation and writing the initial draft. M.Q.F. was responsible for the methodology, data collection and data analysis. M.A.P. was responsible for the methodology, data analysis, validation, writing revisions and student supervision.

\section{References}

1. Waring MJ, Arrowsmith J, Leach AR, Leeson PD, Mandrell S, Owen $\mathrm{RM}$, et al. An analysis of the attrition of drug candidates from four major pharmaceutical companies. Nat Rev Drug Discov. 2015;14:475-486. https:// doi.org/10.1038/nrd4609
2. McGovern SL, Caselli E, Grigorieff N, Schoichet BK. A common mechanism underlying promiscuous inhibitors from virtual and high-throughput screening. J Med Chem. 2002;45:1712-1722. https://doi.org/10.1021/jm010533y

3. McGovern SL, Helfand BT, Feng B, Shoichet BK. A specific mechanism of nonspecific inhibition. J Med Chem. 2003;46:4265-4272. https://doi. org/10.1021/jm030266r

4. Baell J, Walters MA. Chemistry: Chemical con artists foil drug discovery. Nature. 2014;513(7519):481-483. https://doi.org/10.1038/513481a

5. Trader DJ, Simanski S, Dickson P, Kodadek T. Establishment of a suite of assays that support the discovery of proteasome stimulators. Biochim Biophys Acta. 2017;1861(4):892-899. https://doi.org/10.1016/j.bbagen.2017.01.003

6. Cao JJ, Forrest C, Zhang X. A screen of the NIH Clinical Collection small molecule library identifies potential anti-coronavirus drugs. Antiviral Res. 2015;114:1-10. https://doi.org/10.1016/j.antiviral.2014.11.010

7. Bushman FD, Engelman A, Palmer I, Wingfield P, Craigie R. Domains of the integrase protein of human immunodeficiency virus type 1 responsible for polynucleotidyl transfer and zinc binding. Proc Natl Acad Sci USA. 1993;90:3428-3432. https://doi.org/10.1073/pnas.90.8.3428

8. Craigie R, Hickman AB, Engelman A. Integrase. In: Karn J, editor. HIV. Volume 2: A practical approach. Oxford: Oxford University Press; 1995. p. 53-71. 
9. Harrison AT, Kriel FH, Papathanasopoulos MA, Mosebi S, Abrahams S, Hewer $\mathrm{R}$. The evaluation of statins as potential inhibitors of the LEDGF/p75 - HIV-1 integrase interaction. Chem Biol Drug Des. 2014;85(3):290-295. https://doi. org/10.1111/cbdd. 12384

10. Grobler JA, Stillmock KA, Hazuda DJ. Scintillation proximity assays for mechanistic and pharmacological analyses of HIV-1 integration. Methods. 2009;49(4):249-253. https://doi.org/10.1016/j.ymeth.2009.03.002

11. Hazuda DJ, Hastings JC, Wolfe AL, Emini EA. A novel assay for the DNA strandtransfer reaction of HIV-1 integrase. Nucleic Acids Res. 1994;22:1121-1122. https://doi.org/10.1093/nar/22.6.1121

12. Harada S, Koyanagi Y, Yamamoto N. Infection of HTLV-III/LAV in HTLV-Icarrying cells MT-2 and MT-4 and application in a plaque assay. Science. 1985;229:563-566. https://doi.org/10.1126/science.2992081

13. Larder BA, Darby G, Richman DD. HIV with reduced sensitivity to zidovudine (AZT) isolated during prolonged therapy. Science. 1989;243:1731-1734. https://doi.org/10.1126/science.2467383

14. Pauwels R, De Clercq E, Desmyter J, Balzarini J, Goubau P, Herdewijn $P$, et al. Sensitive and rapid assay on MT-4 cells for detection of antiviral compounds against the AIDS virus. J Virol Meth. 1987;16:171-185. https:// doi.org/10.1016/0166-0934(87)90002-4
15. Mphahlele MK, Papathanasopoulos MA, Cinellu MA, Coyanis EM, Mosebi $\mathrm{S}$, Traut T, et al. Modification of HIV-1 reverse transcriptase and integrase activity by gold(III) complexes in direct biochemical assays. Bioorg Med Chem. 2012;20:401-407. https://doi.org/10.1016/j.bmc.2011.10.072

16. Ingolfsson $\mathrm{HI}$, Thukur P, Herold KF, Hobart EA, Ramsey NB, Periole X, et al. Phytochemicals perturb membranes and promiscuously alter protein function. ACS Chem Biol. 2014;9(8):1788-1798. https://doi.org/10.1021/ cb500086e

17. ClinicalTrials.gov. A service of the U.S. National Institutes of Health [homepage on the Internet]. No date [cited 2017 Mar 20]. Available from: https://clinicaltrials.gov

18. Malet I, Delelis O, Valantin MA, Montes B, Soulie C, Wirden M, et al. Mutations associated with failure of raltegravir treatment affect integrase sensitivity to the inhibitor in vitro. Antimicrob Agents Chemother. 2008;52(4):1351-1358. https://doi.org/10.1128/AAC.01228-07

19. Witmer M, Danovich R. Selection and analysis of HIV-1 integrase strand transfer inhibitor resistant mutant viruses. Methods. 2009;47:277-282. https://doi.org/10.1016/j.ymeth.2009.02.025

20. Ravishankar S, Kumar VP, Chandrakala B, Jha RK, Solapure SM, De Sousa SM. Scintillation proximity assay for inhibitors of Escherichia coli MurG and, optionally, MraY. Antimicrob Agents Chemother. 2005;49:1410-1418. https://doi.org/10.1128/AAC.49.4.1410-1418.2005 